\title{
Restorative tips for primary care (part 1)
}

\author{
By R. J. R. Smith, ${ }^{1}$ G. E. Bryce ${ }^{2}$ and E. McColl ${ }^{3}$
}

$\mathrm{T}$ he British Society of Restorative Dentistry (BSRD) defines restorative dentistry as the study, examination and treatment of diseases of the oral cavity, the teeth and their supporting structures. Restorative dentistry includes the dental monospecialties of endodontics, periodontics and prosthodontics (including implantology), and its foundation is based upon how these interact in the management of cases requiring multifaceted care. ${ }^{1}$

It is impossible to cover the extensive scope of all the domains that encompass restorative dentistry within the limits of this series of articles. Subsequently, the challenges that we discuss are those that we have experienced from our own clinical trial and error, questions that have been frequently posed, or observations of failing treatments. These tips are experiential in many respects and, whilst we are fully cognisant of the time pressures and fiscal demands dental teams face, we hope that the advice given can not only improve outcomes for patients but also help hard-pressed clinicians to manage the clinical challenges they face daily.

\section{Choosing a posterior restorative material}

a. Amalgam restorations can be selected for use in posterior teeth where isolation is suboptimal, and can be especially useful in the following situations: i) the restoration of partially erupted third molars; ii) the restoration of distal cervical caries lesions in mandibular second molars, where impacted third molars have been extracted and in conjunction with a deep circumferential matrix band

b. Glass-ionomer cement (GIC), with its inherent ability to bond to tooth tissue, makes it particularly useful for management of Class $\mathrm{V}$ cavities associated with non-carious tooth surface loss, especially where such lesions are associated with symptoms of dentine (hyper)sensitivity

c. Composite can be used for all other definitive restorations.

\section{Working with composite}

a. Compared to total etch techniques, a selective etching technique can increase bond strengths to dentine ${ }^{2}$ whilst reducing the risk of post-operative sensitivity and other pulprelated complications. In practice, this could be achieved via the following protocol: i) selective placement of etch on the enamel of the cavity margin (a periodontal probe can be used to place the etch); ii) rinsing off the etch after 20 seconds and drying (not desiccating); iii) application of a self-etch adhesive (eg Scotchbond Universal Adhesive, 3M ESPE, St Paul, USA), to both the dentine and enamel with microbrush; iv) rubbing the adhesive into the dentine for up to 20 seconds, as rubbing has been shown to increase the bond strength; ${ }^{3} \mathrm{v}$ ) incremental placement and curing of composite prior to finishing procedures

b. Warming composite increases flowability and aids adaptability of the material. ${ }^{4} \mathrm{~A}$ variety of appliances have been specifically manufactured for composite warming but alternate approaches include the use of coffee-cup warmers, or placing the composite capsules in a sealed bag within a bowl filled with hot water. Nonstick contouring instruments (eg OptraSculpt, Ivoclar Vivadent, Amherst, USA) and brushes are ideal for layering and shaping.

\section{Placement of composite within the sub-gingival cavity zone}

a. Moisture control. Gingival bleeding control can be assisted with intra-papillary infiltrations of a local anaesthetic (LA) solution containing a vasoconstrictor. Sub-gingival placement of a retraction cord soaked in a vasoconstrictor containing LA can be used as an adjunct in cases where gingival bleeding is particularly troublesome. Rubber dam should be placed to control saliva. A suitable matrix should then be placed and wedged, ensuring that the matrix closely approximates the cavity margins

b. Following etching and application of a dental adhesive, an increment of composite should first be placed, adapted and cured in the deep sub-marginal aspect of the cavity; this will tend to further stabilise the matrix prior to addition of further layers 
$\varangle$ b. When restoring a Class II cavity, the tooth undergoing the procedure and (where present) the tooth adjacent to the cavity should be isolated. The more posterior of the teeth will need a clamp to resist the tension in the dam. The dam can be secured on the more anterior tooth by either another clamp, floss ligature or elastic stabilising cord (eg Wedjets, Coltene/ Whaledent, Cuyahoga Falls, USA)

C. Placement of unbonded composite onto the outside surface of the matrix can also help stop the rubber dam slipping, whilst also improving moisture control.

\section{Polishing composites - reducing the risk of staining?}

a. The oxygen-inhibited layer on the external surface of newly placed composite resin restoration contains unpolymerised monomer, which is liable to absorb stain. ${ }^{6}$ Polishing to remove this layer can be challenging in hard-to-reach areas, such as fissures and interproximal areas

b. Coating the final layer of composite, prior to curing, with glycerol delivered with a microbrush reduces this problem. Most polishing systems on the market are effective if the entirety of the recommended polishing protocol is followed.

\section{Repairing/replacing fractured composite}

a. Firstly, carry out an assessment to determine the type of the composite fracture (eg adhesive vs cohesive failure), and identify and manage any contributing factors, such as: caries, an occlusal interference, or parafunctional habits

b. If the failure is due to loss of adhesion, then the composite will need to be replaced. The composite bulk can be removed using a high-speed handpiece, and then a slow-speed handpiece and composite-removing bur used to carefully remove the tooth surface adhered layer. Resin tags from the tooth surface can be removed using a sandblaster, with the oral cavity shielded from the tooth using rubber dam.

In the event that the cohesive fracture and the remainder of the restoration is assessed to be satisfactory with no secondary caries or voids, then the restoration may be repaired. The surface layer of composite should be removed with a bur and the surface further roughened via sandblasting under rubber dam, prior to etching, bonding and re-restoring with composite.

\section{In part 2, we will expand further on tips relating to fixed} prosthodontics and endodontics.

\section{References}

1. The British Society of Restorative Dentistry. Homepage. Available at: https://www.bsrd. org.uk/Index.aspx (accessed January 2022).

2. Goracci C, Rengo C, Eusepi L, Juloski J, Vichi A, Ferrari M. Influence of selective enamel etching on the bonding effectiveness of a new all-in-one adhesive. Am J Dent 2013; 26: 99-104.

3. Dal-Bianco K, Pellizzaro A, Patzlaft R, de Oliveira Bauer J R, Loguercio A D, Reis A. Effects of moisture degree and rubbing action on the immediate resin-dentin bond strength. Dent Mater 2006; 22: 1150-1156.

4. Deb S, Di Silvio L, Mackler H E, Millar B J. Pre-warming of dental composites. Dent Mater 2011; 27: 51-59.

5. Van Dijken J W, Kieri C, Carlen M. Longevity of extensive class II open-sandwich restorations with a resin-modified glass-ionomer cement. J Dent Res 1999; 78: 1319-1325

6. Park S H, Noh B D, Ahn H J, Kim H K. Celluloid strip-finished versus polished composite surface: difference in surface discoloration in microhybrid composites. J Oral Rehabil 2004; 31: 62-66.

\section{Honours, awards, appointments}

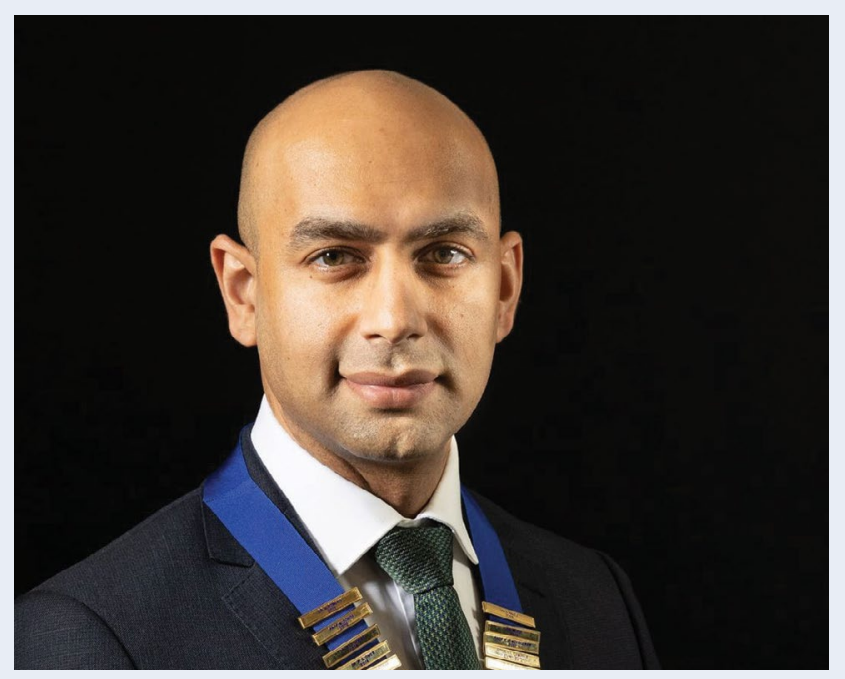

\section{BSP President}

Dr Rajan Nansi (pictured) has been appointed President of the British Society of Periodontology and Implant Dentistry (BSP) for 2022, taking over from Professor Mark Ide.

As a long-serving member of the BSP, Rajan is a dedicated supporter of its work and was instrumental in setting up its Early Careers Group.

Rajan is based at his practice RESTORE Dental Specialists in Worcestershire.

\section{Professor of Dental Public Health}

Professor Manu Raj Mathur joined Queen Mary University of London's Centre for Dental Public Health and Primary Care on 1 February 2022 as Professor of Dental Public Health.

Professor Mathur is a dental surgeon with more than 15 years of global experience of working in different health systems. In his new role, he will contribute to research and implementation activities of Barts Centre for Squamous Cancer (BCSC), a new centre of excellence dedicated to improving detection, treatment and quality of life for patients with squamous cancer funded by Bart's Charity.

\section{Ellison-Cliffe Travelling Fellowship}

Daniel Van Gijn, an ST7 specialist registrar in oral and maxillofacial surgery at the Royal Surrey NHS Foundation Trust, is one of five successful applicants for the Royal Society of Medicine (RSM) Ellison-Cliffe Travelling Fellowships.

With the support of the travelling fellowship, he will be spending a year in Australia at the Chris O'Brien Lifehouse (COBLH). Daniel has a fellowship in head and neck surgery and post-ablative microvascular reconstruction at the COBLH in Sydney.

Daniel recently published the new Oxford Handbook of Head and Neck Anatomy. 